\title{
Global Optimization of Robotic Grasps
}

\author{
Carlos Rosales \\ Institut de Robòtica i Informàtica Industrial, CSIC-UPC \\ Institut d'Organizació i Control de Sistemes Industrials, UPC \\ Barcelona, Spain \\ Email: crosales@iri.upc.edu
}

\author{
Josep M. Porta and Lluís Ros \\ Institut de Robòtica i Informàtica Industrial, CSIC-UPC \\ Barcelona, Spain \\ Email: \{ros,porta\}@iri.upc.edu
}

\begin{abstract}
This paper presents a procedure to optimize the quality of robotic grasps for objects that need to be held and manipulated in a specific way, characterized by a number of tight contact constraints. The main difficulties of the problem include that the set of feasible grasps is a manifold implicitly defined by a system of non-linear equations, the high dimension of this manifold, and the multi-modal nature of typical grasp quality indices, which make local optimization methods get trapped into local extrema. The proposed procedure finds a way around these difficulties by focussing the exploration on a relevant subset of grasps of lower dimension, which is traced out exhaustively using higher-dimensional continuation techniques. Using these techniques, a detailed atlas of the subset is obtained, on which the highest quality grasp according to any desired criterion can be readily identified. Experiments on a 3-finger planar hand and on the Schunk anthropomorphic hand validate the approach.
\end{abstract}

\section{INTRODUCTION}

The study of how to grasp and manipulate objects with anthropomorphic hands is fundamental if robots are ever to replace humans in dangerous or repetitive tasks, or to cooperate with them in everyday activities. In many cases, the objects are designed to be held and manipulated in a specific way, characterized by a number of tight contact constraints [2, 9, 8, 19, 18]. In such cases, a grasp planner must be able to generate, not only a grasp satisfying the contact constraints, but one of sufficient quality, guaranteeing that the task will be successfully accomplished. For instance, Fig. 1 shows two different grasps of a can during drink service. While the can easily escapes from the hand in the first grasp, it is firmly held in the second one, even in the presence of external disturbances. Since the quality of a grasp may be defined according to a variety of criteria, including force-closure [6], manipulability [1], or any other criterion particular to the task to be performed [13], the grasp planner should ideally make as few assumptions as possible on the continuity or smoothness of the quality measure adopted.

When the object can be grasped in an arbitrary way, a grasp can be optimized in a generate-and-test fashion, initially relaxing the contact constraints [3]. The resulting search space is of high dimension after such relaxation as it coincides with the configuration space of the hand. Therefore, dimensionreduction simplifications based on principal hand motions [21. 3, 20, 7] need to be introduced to define a search space that can be explored in a reasonable time. This space is searched effectively in [3], for example, using simulated annealing. The

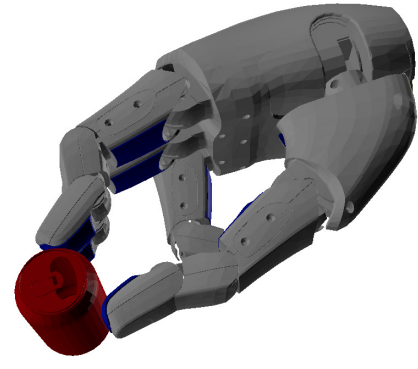

(a)

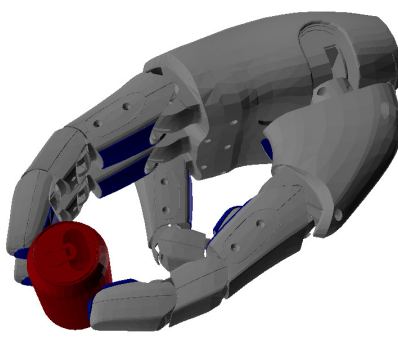

(b)
Fig. 1. Two grasps of the same object. (a) A low quality grasp where the hand can not compensate external disturbances. (b) A grasp where the hand firmly holds the object.

generated solutions, however, do not necessarily correspond to hand configurations in contact with the object, and thus they need to be evaluated with so-called pre-grasp quality indices. Unfortunately, a good pre-grasp does not always result in a high-quality grasp, once the required contact constraints are finally enforced.

The problem is harder if, as considered in this paper, the object has to be grasped and manipulated while respecting a number of tight contact constraints with the hand. In this case, the space of feasible grasps is a complex manifold, implicitly defined by a system of nonlinear equations that express all assembly and contact constraints of the hand-object system. Typically, such manifold is not parametrizable and, thus, it can not be effectively explored with randomized techniques like [3] since the probability of obtaining a valid grasp using a generate-and-test strategy is very low. Moreover, local methods departing from a given configuration [15] are not likely to find a globally optimal grasp, because grasp quality indices are highly non-linear and present local extrema.

This paper proposes a grasp optimization procedure aimed at circumventing the aforesaid limitations. The procedure entails characterizing the manifold of feasible grasps as a system of equations to be fulfilled (Section II), then extending this system with meaningful equations to reduce the dimension of its solution set (Section III), and finally performing an exhaustive search over a point grid discretizing such set at a desired resolution, to determine the highest-quality grasp attainable on the grid (Section IV]. The grid is derived by resorting to recently-developed techniques for higher-dimensional contin- 
uation [11], which are able to compute exhaustive chart-based representations of implicitly-defined manifolds in reasonable times, for moderate dimensions. The method can be applied to optimize a grasp under any desired criterion, because it only requires the evaluation of the adopted quality measure on selected points, without making particular assumptions on the mathematical properties of the measure. The approach has been validated on simple and complex grasping devices (Section $\mathrm{V}$ ) using several quality indices.

\section{Feasible Grasp Characterization}

Robotic hands are complex mechanical systems involving multiple bodies articulated through lower-pair joints (Fig. 2). In many situations, like when serving a drink or manipulating a scalpel, a hand is required to grasp an object in a specific way, characterized by a number of points $\mathbf{q}_{i}$ on the hand, $i=1, \ldots, m$, that need to be placed in contact with corresponding regions $\mathcal{O}_{i}$ on the object, keeping aligned the surface normals to the hand, $\hat{\mathbf{n}}_{i}$, and the object, $\hat{\mathbf{m}}_{i}$, to avoid interpenetrations (Fig. 3). While the hand points may derive from known patterns of static prehension [12], the object regions may be obtained from contact region delimitation algorithms [14, 22, 16, 17], and the assignment of hand points to object regions may be done using representative points on the regions [24, 5]. As shown next, the set of feasible grasps, i.e., those fulfilling such constraints, can be characterized by a system of nonlinear equations.

Regardless of the specific formulation adopted, a grasp configuration can be represented by a vector $\mathbf{x}=\left[\mathbf{x}_{h}, \mathbf{x}_{o}, \mathbf{x}_{c}\right]$ of $n$ generalized coordinates, where $\mathbf{x}_{h}$ and $\mathbf{x}_{o}$ encompass the configuration variables of the hand and the object, respectively, and $\mathbf{x}_{c}$ encompasses contact-related variables. Without loss of generality, we will assume that the absolute reference frame is attached to the palm of the hand, so that its pose variables do not intervene in $\mathbf{x}$.

The $\mathbf{x}$ variables are subject to a number of constraints. A first set of equations

$$
\mathbf{H}\left(\mathbf{x}_{h}\right)=\mathbf{0}
$$

enforces $\mathbf{x}_{h}$ to be a valid hand configuration, i.e., one respecting the assembly constraints imposed by the joints (usually revolute or universal) on the various bodies they connect (the palm and the several finger phalanges). Note that Eq. (1) is not necessary if the $\mathbf{x}_{h}$ coordinates are independent, as it happens for instance when choosing joint angles to represent a configuration [4]. In our case, however, we use the dependent coordinates proposed in [18] because they yield equations of a simple structure, which has proved to be beneficial in the context of continuation methods [23]. Thus, Eq. (1) encompasses the constraints relating such coordinates. In particular, this formulation encodes the 6 degrees of freedom a body with 12 variables providing its position vector and rotation matrix. Therefore, in addition to the joint assembly equations, Eq. (1) includes constraints to enforce the 12-tuple for each body to be a member of $S E(3)$. In a similar way, a second

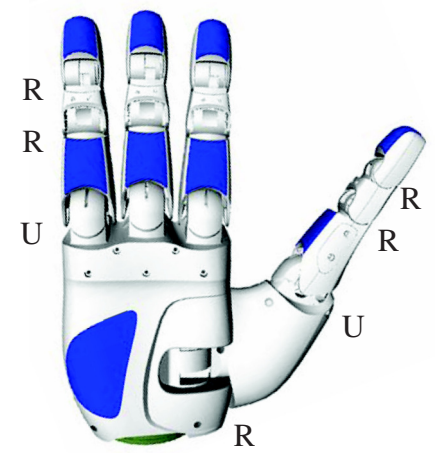

Fig. 2. Kinematic structure of the Schunk anthropomorphic hand. R stands for revolute joint and $\mathrm{U}$ for universal.

set of equations,

$$
\mathbf{L}\left(\mathbf{x}_{o}\right)=\mathbf{0},
$$

constrains $\mathbf{x}_{o}$ to be a member of $S E(3)$. Finally, a third set of equations formulates the contact constraints between the hand and the object. To this end, we assume that each region $\mathcal{O}_{i}$ is specified as a parametrized patch, i.e., as a smooth function of the form

$$
\mathbf{o}_{i}=\mathbf{O}_{i}\left(u_{i}, v_{i}, \mathbf{x}_{o}\right)
$$

providing the absolute coordinates of a point $\mathbf{o}_{i}=\left(x_{i}, y_{i}, z_{i}\right)$ in the patch, in terms of two scalar parameters, $u_{i}$ and $v_{i}$, bound to lie within some interval, and of the object pose $\mathbf{x}_{o}$. Analogously, the normal to any point in this patch is assumed to be given as a function

$$
\hat{\mathbf{m}}_{i}=\mathbf{M}_{i}\left(u_{i}, v_{i}, \mathbf{x}_{o}\right) .
$$

Both $\mathbf{O}_{i}\left(u_{i}, v_{i}, \mathbf{x}_{o}\right)$ and $\mathbf{M}_{i}\left(u_{i}, v_{i}, \mathbf{x}_{o}\right)$ can be defined using, for instance, Bézier patches [18]. Note that since the forward kinematic map of the hand allows writing $\mathbf{q}_{i}$ and $\hat{\mathbf{n}}_{i}$ as functions of the hand variables

$$
\begin{aligned}
\mathbf{q}_{i} & =\mathbf{K}_{i}\left(\mathbf{x}_{h}\right), \\
\hat{\mathbf{n}}_{i} & =\mathbf{N}_{i}\left(\mathbf{x}_{h}\right),
\end{aligned}
$$

the contact of $\mathbf{q}_{i}$ with $\mathbf{o}_{i}$ can be expressed by setting

$$
\begin{aligned}
\mathbf{q}_{i} & =\mathbf{o}_{i}, \\
\hat{\mathbf{m}}_{i} & =-\hat{\mathbf{n}}_{i} .
\end{aligned}
$$

In sum, thus, the set of feasible grasps is the set $\mathcal{F}$ of points $\mathrm{x} \in \mathbb{R}^{n}$ satisfying the system

$$
\mathbf{F}(\mathbf{x})=\mathbf{0}
$$

formed by Eqs. (1) and (2), and Eqs. (3) to (8) for $i=$ $1, \ldots, m$. We will here assume that $\mathbf{F}(\mathbf{x})$ is a smooth function, whose Jacobian is full rank at all points satisfying Eq. (9), which is the common situation in practice. Thus, $\mathcal{F}$ will be a smooth manifold in our case. 


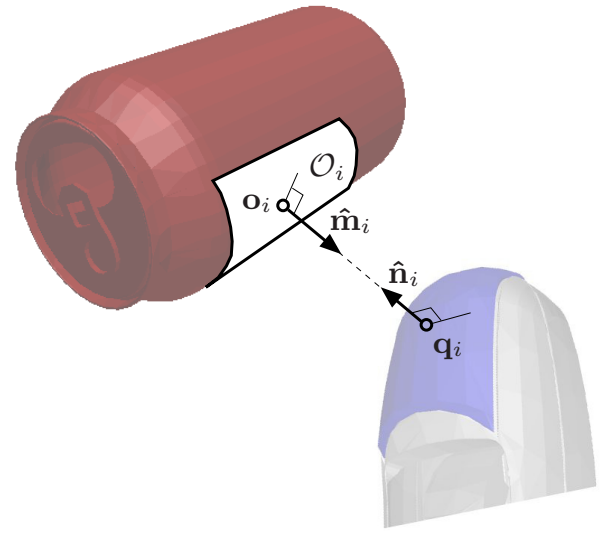

Fig. 3. Elements intervening in the $i$-th contact constraint. Points $\mathbf{q}_{i}$ and $\mathbf{o}_{i} \in \mathcal{O}_{i}$ must coincide, with the normals on such points, $\hat{\mathbf{n}}_{i}$ and $\hat{\mathbf{m}}_{i}$, aligned.

\section{DIMENSION REDUCTION}

For a realistic hand and contact model, the dimension of $\mathcal{F}$ is very large, which hinders the efficient exploration of this space independently of the methodology adopted. In the context of grasping, however, studies on the human behavior suggest that humans do not use all the degrees of freedom of the hand independently, but in a coordinated way [21]. Following this idea, anthropomorphic hands are usually controlled using so-called principal hand motions (also called hand postural synergies [21, 7], eigengrasps [3], or principal motion directions [20]), where few coordinated motions are used to account for the overall motion capability of the hand. By taking principal hand motions into consideration, the search of a good grasp can be narrowed to a subset $\mathcal{R} \subset \mathcal{F}$ of lower dimension, thus speeding up the exploration.

Principal hand motions are computed via linear dimensionreduction techniques on a predetermined set of hand configurations $\mathbf{X}_{h}=\left\{\mathbf{x}_{h}^{i} \mid i=1, \ldots, z\right\}$. Let $\overline{\mathbf{x}}_{h}$ be the average of the configurations in $\mathbf{X}_{h}$, and let $\mathbf{T}$ be a $h \times z$ matrix where each column $t_{i}$ is $\mathbf{x}_{h}^{i}-\overline{\mathbf{x}}_{h}$. The principal component analysis of $\mathbf{T}$ can be performed by diagonalizing the covariance of $\mathbf{T}$ as

$$
\mathbf{T} \mathbf{T}^{\top}=\mathbf{E} \mathbf{S}^{2} \mathbf{E}^{\top}
$$

The $h \times h$ orthonormal matrix $\mathbf{E}$ gives the directions of variance of the data, and the diagonal matrix $\mathbf{S}^{2}$ is the variance in each one of these directions, sorted in decreasing magnitude. The set $\mathcal{E}$ of the $p$ principal hand motions is spanned by the first $p$ columns of $\mathbf{E}$, and the matrix $\mathbf{E}_{s}$, including the rest of columns of $\mathbf{E}$, spans the remaining $s=h-p$ hand motions. Therefore, Eq. (9), together with

$$
\mathbf{E}_{s}^{\top}\left(\mathbf{x}_{h}-\overline{\mathbf{x}}_{h}\right)=\mathbf{0},
$$

defines the system

$$
\mathbf{R}(\mathbf{x})=\mathbf{0} \text {, }
$$

characterizing the set $\mathcal{R}=\mathcal{F} \cap \mathcal{E}$ of relevant grasps.

For a given $t$, the dimension of $\mathcal{F}$, and a given $k$, the desired dimension of $\mathcal{R}$, the number of additional constraints

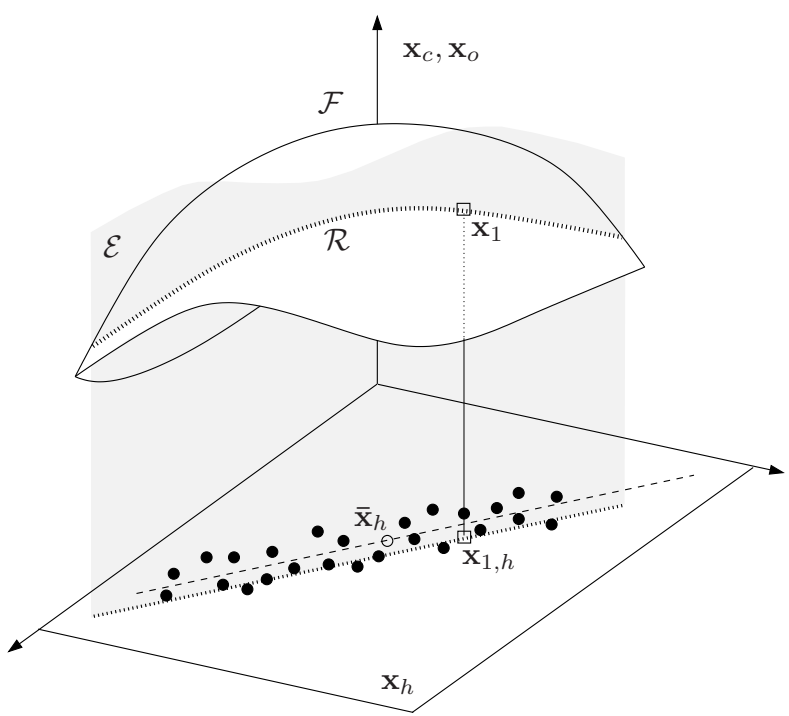

Fig. 4. Schematic representation of all the elements involved in the optimization framework presented in this paper. See the text for details.

in Eq. (10) to add to Eq. (9) must be

$$
s=t-k
$$

Note that $s$ must be smaller than $h$, which limits the amount of dimension reduction introduced by the use of principal hand motions. As we will see, this is not an issue in practice, since, due to the presence of the contact constraints, the amount of dimension reduction to be introduced is moderate in all cases.

Care must be taken, however, because the introduction of principal hand motions might lead to an empty set $\mathcal{R}$. In order to avoid this issue, and to guarantee the intersection of $\mathcal{F}$ and $\mathcal{E}$, we approximate Eq. (10) by

$$
\mathbf{E}_{s}^{\top}\left(\mathbf{x}_{h}-\mathbf{x}_{1, h}\right)=\mathbf{0}
$$

where $\mathbf{x}_{1, h}$ corresponds to the hand parameters of an initial feasible grasp, $\mathbf{x}_{1} \in \mathcal{F}$, which may be obtained using grasp synthesis techniques [2, 18]. This approximation ensures that the hand always conforms to the object surface because $\mathcal{R}$ includes at least one feasible grasp, $\mathbf{x}_{1}$. However, in general, $\mathcal{R}$ is large since the difference of using the $\mathbf{x}_{1, h}$ instead of $\overline{\mathbf{x}}_{h}$ is small. This is due to the fact that the components of any hand configuration along $\mathbf{E}_{s}$ are typically small and, thus, $\mathbf{x}_{1, h}$ is usually close to the original set of principal hand motions.

Figure 4 summarizes the different elements involved in the approach. $\mathcal{F}$ is the set of feasible grasps defined in the space of $\mathbf{x}_{h}, \mathbf{x}_{o}$, and $\mathbf{x}_{c}$. In this representation, the configurations in $\mathbf{X}_{h}$ are shown as black dots, the white dot is their average, $\overline{\mathbf{x}}_{h}$, and the original set of principal hand motions is shown as a dashed line in the $\mathbf{x}_{h}$ plane. The latter set is approximated by a line through $\mathbf{x}_{1, h}$, shown dotted in the figure, which, when extended to the whole space, generates the linear space $\mathcal{E}$. Finally, the set $\mathcal{R}=\mathcal{F} \cap \mathcal{E}$ is the space where the grasp optimization is to be performed. 


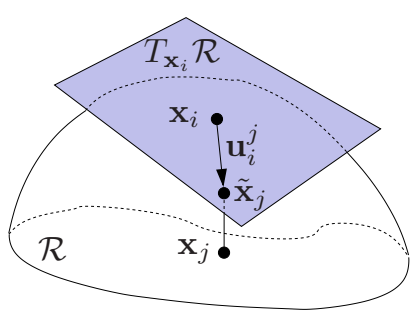

(a)

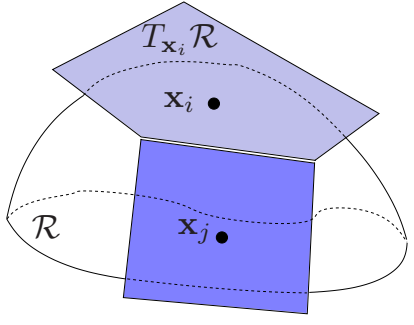

(b)
Fig. 5. The higher-dimensional continuation method applied to a twodimensional manifold in $3 \mathrm{D}$ space. (a) A point $\mathbf{x}_{j}$ on $\mathcal{R}$ can be obtained by orthogonally projecting a point $\tilde{\mathbf{x}}_{j}$ on $T_{\mathbf{x}_{i}} \mathcal{R}$. (b) If a new chart is defined at $\mathbf{x}_{j}$, it must be properly coordinated with the chart at $\mathbf{x}_{i}$ so that their projections smoothly covers the manifold.

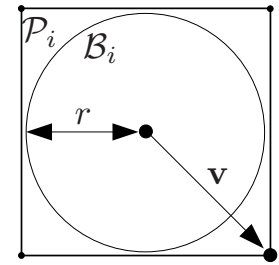

(a)

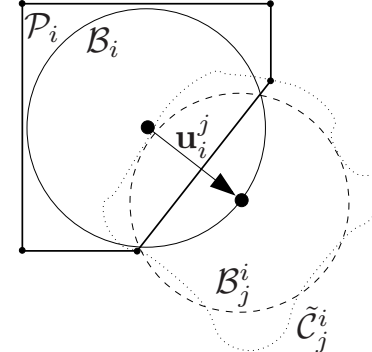

(b)
Fig. 6. Polytope-based chart construction. (a) The validity area for chart $\mathcal{C}_{i}$, $\mathcal{P}_{i}$, is a box including a ball of radius $r$ around $x_{i}$. (b) $\mathcal{P}_{i}$ is refined using a ball $\mathcal{B}_{j}^{i}$ that approximates $\tilde{\mathcal{C}}_{j}^{i}$, the projection on $\mathcal{C}_{i}$ of the part of the manifold covered by $\mathcal{C}_{j}$.

\section{GRASP QuALity OPTIMIZATION}

In this paper, the optimal grasp is computed by obtaining an atlas of $\mathcal{R}$. An atlas is composed by a collection of charts where each chart $\mathcal{C}_{i}$ defines a map from $\mathbb{R}^{k}$ to a portion of $\mathcal{R}$ around a given point $\mathbf{x}_{i} \in \mathbb{R}^{n}$. This atlas will allow enumerating a representative collection of grasps in $\mathcal{R}$, on which any given quality index can be evaluated. The atlas will be computed using the higher-dimensional continuation method proposed in [10]. This method defines the map for chart $\mathcal{C}_{i}$ using $\boldsymbol{\Phi}_{i}$, an orthonormal basis of $T_{\mathbf{x}_{i}} \mathcal{R}$, the $k$ dimensional tangent space of $\mathcal{R}$ at $\mathbf{x}_{i}$. The map is defined by first selecting a $k$-dimensional vector $\mathbf{u}_{i}^{j}$ of parameters (Fig. 51), which is used to generate a point $\tilde{\mathbf{x}}_{j} \in \mathbb{R}^{n}$ in the neighborhood of $\mathbf{x}_{i}$ as

$$
\tilde{\mathbf{x}}_{j}=\mathbf{x}_{i}+\boldsymbol{\Phi}_{i} \mathbf{u}_{i}^{j}
$$

Then, a point $\mathbf{x}_{j}$ on $\mathcal{R}$ is computed by orthogonally projecting $\tilde{\mathbf{x}}_{j}$. This projection is obtained by solving

$$
\begin{aligned}
\mathbf{R}\left(\mathbf{x}_{j}\right) & =\mathbf{0}, \\
\boldsymbol{\Phi}^{\top}\left(\mathbf{x}_{j}-\tilde{\mathbf{x}}_{j}\right) & =\mathbf{0},
\end{aligned}
$$

using a Newton process initialized at $\tilde{\mathbf{x}}_{j}$.

Each point on the manifold is the potential center of a new chart (see Fig. 5b). Henderson [10] introduces a method to
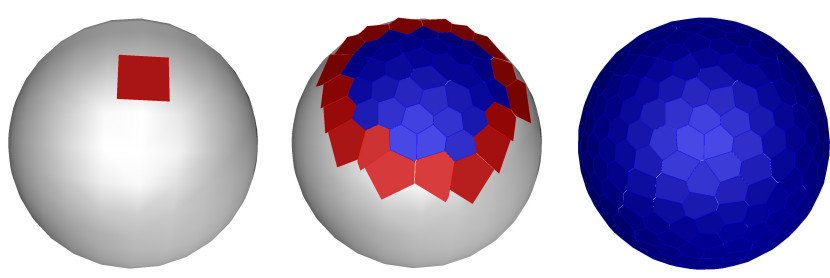

Fig. 7. Three stages in the construction of an atlas over a sphere. Red and blue polygons represent charts under expansion and charts whose domain is already bounded, respectively.

determine how to select the centers for the charts, ensuring a good coverage of the manifold. In his approach, the domain $\mathcal{P}_{i}$ of chart $\mathcal{C}_{i}$ is initialized as a $k$-dimensional hypercube enclosing a ball $\mathcal{B}_{i}$ of radius $r$, both defined in $T_{\mathbf{x}_{i}} \mathcal{R}$, as illustrated in Fig. 6a. A vertex of $\mathcal{P}_{i}$ exterior to $\mathcal{B}_{i}$, with position vector $\mathbf{v}$, is used to generate a point $\tilde{\mathbf{x}}_{j}$, using Eq. (14) with

$$
\mathbf{u}_{i}^{j}=\frac{\alpha}{\|\mathbf{v}\|} \mathbf{v}
$$

where $\alpha$ is initialized to $r$. If the projection from $\tilde{\mathbf{x}}_{j}$ to $\mathcal{R}$ does not converge, or if the new chart $\mathcal{C}_{j}$ at $\mathbf{x}_{j}$ is too far or too different from $\mathcal{C}_{i}$, i.e., if

$$
\left\|\mathbf{x}_{j}-\tilde{\mathbf{x}}_{j}\right\|>\epsilon
$$

or

$$
\left\|\boldsymbol{\Phi}_{i}^{\top} \boldsymbol{\Phi}_{j}\right\|<1-\epsilon,
$$

for a given threshold $\epsilon$, then the new chart is discarded and a new attempt of chart generation is performed with a smaller $\alpha$. This procedure adapts the size of the area covered by each chart to the local curvature of the manifold. When $\mathcal{C}_{j}$ is valid, it is used to refine $\mathcal{P}_{i}$ from the intersection between $\mathcal{B}_{i}$ and $\tilde{\mathcal{C}}_{j}^{i}$, the projection on $T_{\mathbf{x}_{i}} \mathcal{R}$ of the part of the manifold covered by $\mathcal{C}_{j}$. This projection is approximated by a ball $\mathcal{B}_{j}^{i}$ in $T_{\mathbf{x}_{i}} \mathcal{R}$, as shown in Fig. 6p. The intersection of $\mathcal{B}_{i}$ and $\mathcal{B}_{j}^{i}$ defines a new face of $\mathcal{P}_{i}$ that eliminates some of its vertices (in particular $\mathbf{v}$ ) and generates new ones. Similarly, the polytope $\mathcal{P}_{j}$ associated with $\mathcal{C}_{j}$ is cropped using the projection of $\mathcal{C}_{i}$ into $\mathcal{C}_{j}$. When $\mathcal{C}_{i}$ is surrounded by other charts, $\mathcal{P}_{i}$ becomes a convex polytope included in $\mathcal{B}_{i}$, and the domain for $\mathcal{C}_{i}$ gets bounded. When all charts are bounded, the connected component of $\mathcal{R}$ containing the initial point $\mathbf{x}_{1}$ gets fully covered, as shown in Fig. 7

The computational cost of the previous procedure is exponential in $k$ and, therefore, it is only practical to compute an atlas on manifolds of moderate dimension, as it is the case of the manifold $\mathcal{R}$ herein considered.

Once the atlas is computed, we can readily evaluate the quality measure all over $\mathcal{R}$. For simplicity, our implementation only evaluates the quality criterion at the chart center. However, if there were abrupt changes in the quality measure, the chart maps could be used to obtain a denser grid of points, either in particular areas or all over the manifold. Collisions could be considered as well, along with the evaluation of the quality measure, returning the best collision free grasp in the end. 


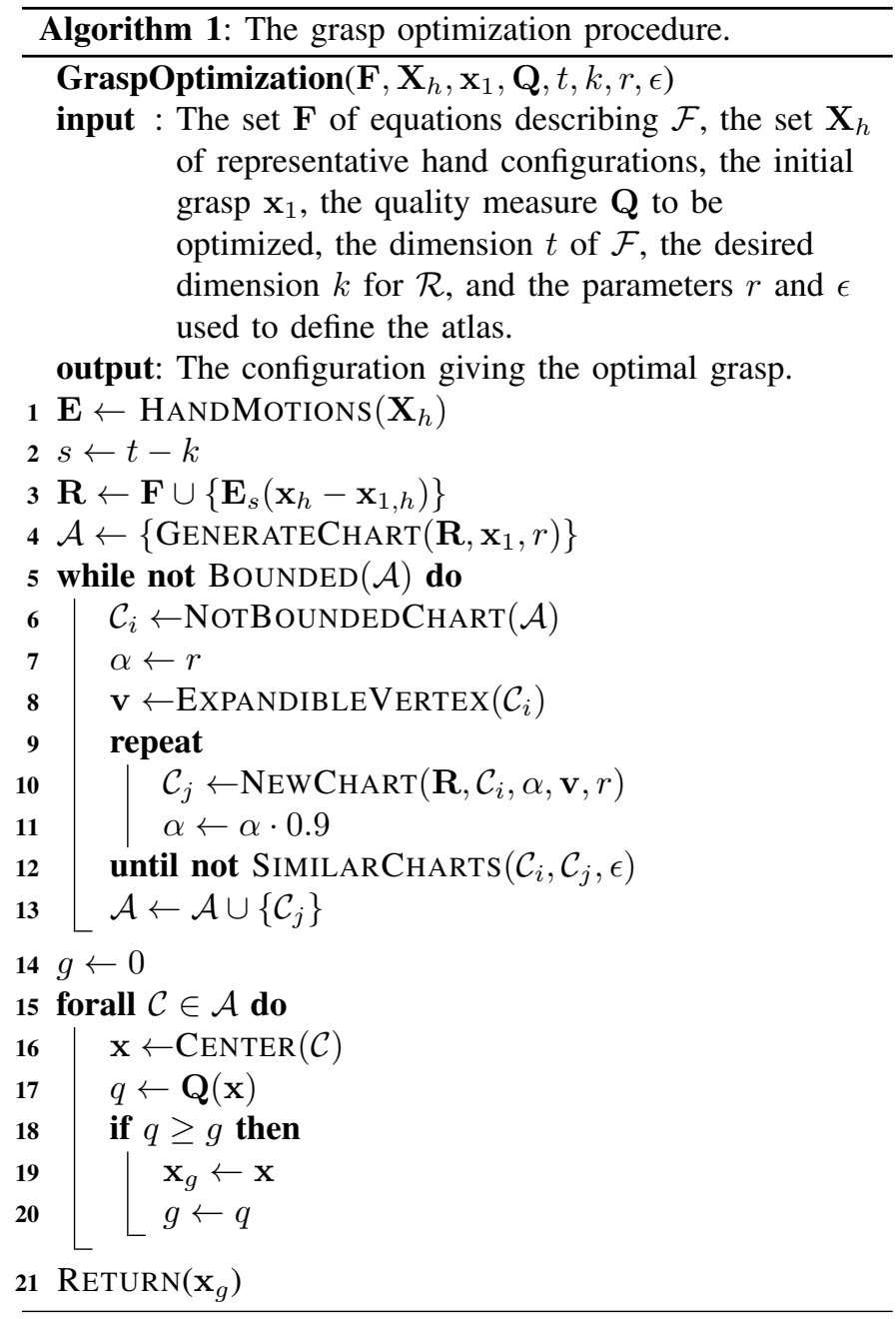

Algorithm 1 summarizes the proposed optimization procedure. The algorithm receives as inputs the set $\mathbf{F}$ of equations describing $\mathcal{F}$, the set $\mathbf{X}_{h}$ of representative hand configurations, the initial grasp $\mathbf{x}_{1}$, the quality measure $\mathbf{Q}$ to be optimized, the dimension $t$ of $\mathcal{F}$, the desired dimension $k$ for $\mathcal{R}$, and the parameters $r$ and $\epsilon$ used to define the atlas. The algorithm determines the hand motions as described in Section IIII (line 1). The number of constraints introduced in $\mathbf{F}$ to obtain $\mathbf{R}$ is computed as a function of $t$ and $k$ (line 2). Then, the atlas is initialized with a chart on $\mathbf{x}_{1}$ (line 4), and the atlas construction proceeds while any of the charts can be extended (lines 5 to 13). The extension of a chart $\mathcal{C}_{i}$ starts by selecting a vertex of $\mathcal{P}_{i}$ not included in $\mathcal{B}_{i}$ (line 8). This vertex is used to generate a new chart $\mathcal{C}_{j}$ (line 10) using Eqs. 114 and (15) to determine its center. If the difference between the new chart and the previous one is too large, according to Eqs. (17) and (18), chart generation is attempted closer to $\mathbf{x}_{i}$. Otherwise, the new chart is added to the atlas, intersecting it with the charts already included in it (line 13). Once the atlas is completed, the quality criterion is evaluated at the chart centers (lines 15 to 20), identifying the point with a larger quality. This point is returned as the optimal grasp (line 21).

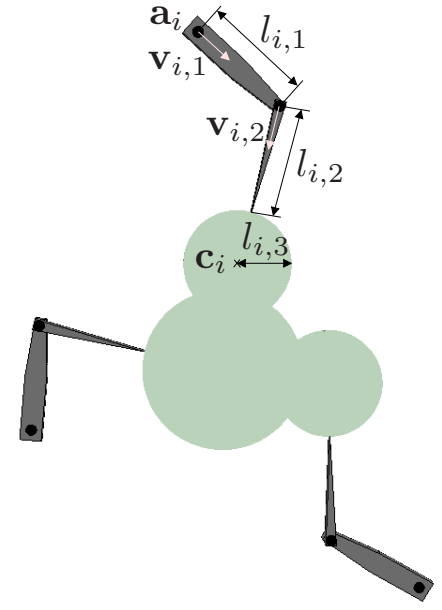

Fig. 8. A simple planar hand with three fingers holding an object. Parameters are only indicated for one finger but apply for the three of them.

\section{EXPERIMENTS AND RESULTS}

For the sake of clarity, we first describe in detail the grasp optimization for a simple example and then summarize the results for the Schunk anthropomorphic hand. All results correspond to an implementation in $\mathrm{C}$ and Matlab, running on an Intel Core 2 Duo at $3 \mathrm{Ghz}$.

Figure 8 shows a planar hand with three fingers and two phalanges per finger holding an object composed of circles. The length and global orientation of the $j$-th phalanx of the $i$-th finger are given by the constant parameter $l_{i, j}$ and the unit vector $\hat{\mathbf{v}}_{i, j} \in \mathbb{R}^{2}$, respectively. The configuration of the hand can be encoded in a simplified form in this case, by the vector $\mathbf{x}_{h}=\left[\hat{\mathbf{v}}_{1,1}, \ldots, \hat{\mathbf{v}}_{3,2}\right]$ subject to the constraints

$$
\left\|\hat{\mathbf{v}}_{i, j}\right\|^{2}=1
$$

for all phalanges. Thus, Eq. (1) is the system formed by Eqs. (19). Since this system contains 6 equations and 12 variables, its solution set will be 6-dimensional, which agrees with the number of degrees of freedom of the hand. The object pose is given by $\mathbf{x}_{o}=\left(\mathbf{t}_{o}, \hat{\mathbf{v}}_{o}\right)$, where $\mathbf{t}_{o}=\left(x_{o}, y_{o}\right)$ and $\hat{\mathbf{v}}_{o}=\left(s_{o}, c_{o}\right)$ are global position and orientation vectors, respectively. Then, Eq. (2) is

$$
\left\|\hat{\mathbf{v}}_{o}\right\|^{2}=1 .
$$

Eq. (5) giving the contact point on each finger is given by

$$
\mathbf{q}_{i}=\mathbf{a}_{i}+l_{i, 1} \hat{\mathbf{v}}_{i, 1}+l_{i, 2} \hat{\mathbf{v}}_{i, 2},
$$

where $\mathbf{a}_{i}$ is the palm anchor point of finger $i$, and Eq. (6) providing the associated normal is simply

$$
\hat{\mathbf{n}}_{i}=\hat{\mathbf{v}}_{i .2} .
$$

In this example, the contact patches reduce to arcs of circumference. Thus, Eq. (3) is

$$
\mathbf{o}_{i}=\left(\mathbf{t}_{o}+\left[\begin{array}{cc}
c_{o} & -s_{o} \\
s_{o} & c_{o}
\end{array}\right] \mathbf{c}_{i}\right)+l_{i, 3} \hat{\mathbf{w}}\left(u_{i}\right)
$$




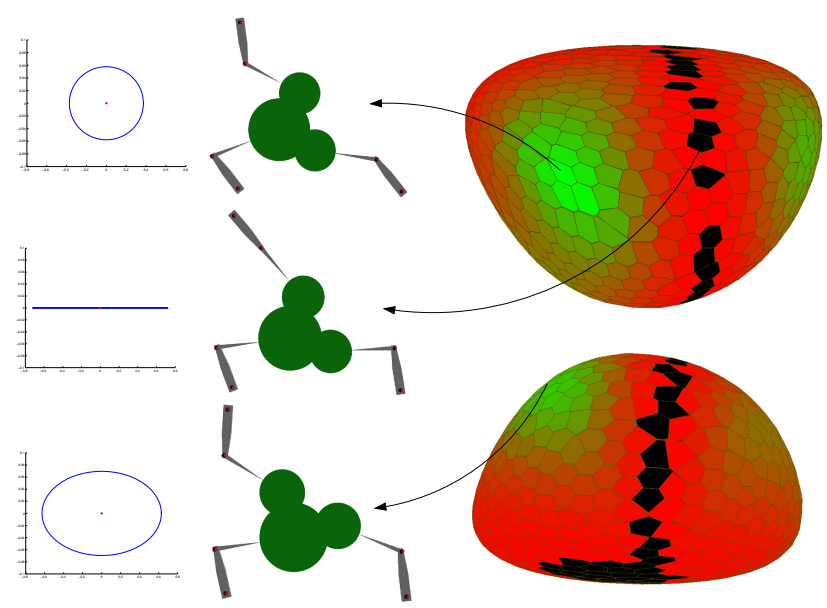

(a)

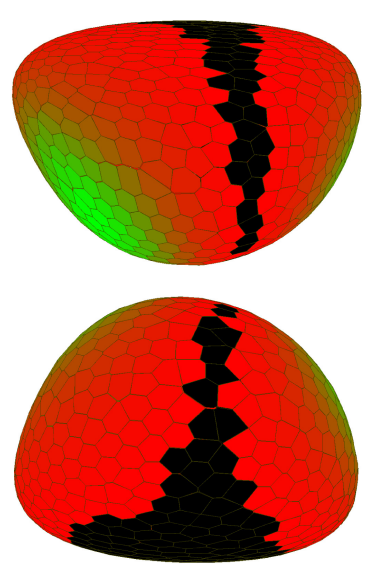

(b)

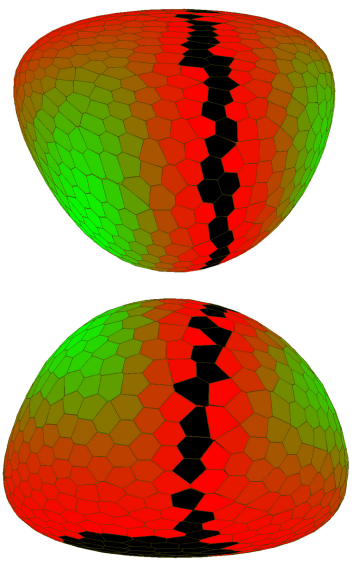

(c)

Fig. 9. Top and bottom views of the atlas over the set $\mathcal{R}$ of relevant feasible grasps, evaluated with three different indices related to the manipulability ellipsoid: (a) the inverse of the condition number, (b) the volume, and (c) the minimum semiaxis length. Green corresponds to configuration with a large value of the corresponding manipulability index, red to configurations with small value, and black to singular configurations. The insets on the left show representative configurations for the hand and the corresponding projection of the manipulability ellipsoid.

where $\mathbf{c}_{i}$ is the center of the circumference in local coordinates of the object, $l_{i, 3}$ is its radius, and

$$
\hat{\mathbf{w}}\left(u_{i}\right)=\left[\begin{array}{c}
\cos u_{i} \\
\sin u_{i}
\end{array}\right],
$$

where $u_{i} \in\left[a_{i}, b_{i}\right]$ is the angular range defining the arc for contact patch $i$. Finally, Eq. (4) giving the normal of the contact patch is

$$
\hat{\mathbf{m}}_{i}=\hat{\mathbf{w}}\left(u_{i}\right) .
$$

Thus, Eq. 9] encompasses Eqs. (19) to (25) in this case. This equation, together with Eq. (13) relative to the principal hand motions, yields Eq. 11] defining $\mathcal{R}$. In this case, the dimension $t$ of $\mathcal{F}$ is 3 . The proposed optimization procedure can be directly applied to problems of this dimension. However, to complete the example and to facilitate the visualization of the results, it is better to reduce the dimension of $\mathcal{R}$ to $k=2$. Thus, using Eq. (12), $s$ must be 1 . In this simulated case, the hand motions are computed from a set $\mathbf{X}_{h}$ of randomlygenerated hand configurations.

Figure 9 shows the results obtained with the proposed method on this example. In the figure, each polygon corresponds to one chart. The total number of charts is about 750 obtained with $r=0.125$ and $\epsilon=0.4$. Since contact patches can be selected to ensure reasonable force-closed grasps [14, 22, 16, 17], the final atlas is used to optimize three indices related to the manipulability ellipsoid of the grasp [1]. These are the inverse of the condition number (the ratio between the smallest and largest semiaxis lengths of the ellipsoid), the volume of the ellipsoid, and its smallest semiaxis length. the manipulability ellipsoid degenerates in a singularity, indicating that the hand can not move the object in some directions. In this case, the manipulability indices are zero. In the plot, green and red correspond to grasps with large and low manipulability, respectively, and black corresponds to singular grasps. A point is considered singular if the corresponding manipulability index is below $10^{-3}$, $10^{-4}$, and $10^{-3}$, respectively. As it can be seen, different indices result in different qualities for the same grasps. The insets on the left show representative grasps for this problem, corresponding to local extrema of the condition number, and the associated manipulability ellipsoid projected on the plane of the largest and smallest semiaxis lengths. The top configuration corresponds to the global optimum obtained. The middle configuration is singular. As it can be seen, one of the fingers is fully extended. This is a well-known singular configuration of the 3RRR planar parallel platform, which is kinematically equivalent to this grasp. Finally, the bottom configuration corresponds to a local maximum. The presence of local extrema makes local search methods not adequate for this problem. In this example, the optimization takes 1.6 seconds, 0.1 to generate the atlas (implemented in C) and 1.5 to evaluate the manipulability criteria (implemented in Matlab).

Figure 10 a shows the results of applying the procedure on the Schunk anthropomorphic hand holding a can with three fingers. Assuming that all joints are independently actuated, this grasp involves 13 degrees of freedom of the hand. In this case, Eq. (1) involves 95 equations and 108 variables and, hence, its solution set is 13-dimensional, as expected. The can is fixed to one of the fingers to avoid repeated solutions caused by its axial symmetry, and the two other fingers are allowed to contact bidimensional patches defined all over the surface of the can. Despite the apparent simplicity of the example, the large extension of the contact patches make this test case a hard one. In this example Eq. 99 involves 126 equations in 134 variables and, hence, $t=8$. To obtain a set $\mathcal{R}$ of dimension $k=2$, which is easier to compute and visualize, $s$ must be set to 6 . By removing only 6 hand motions out 


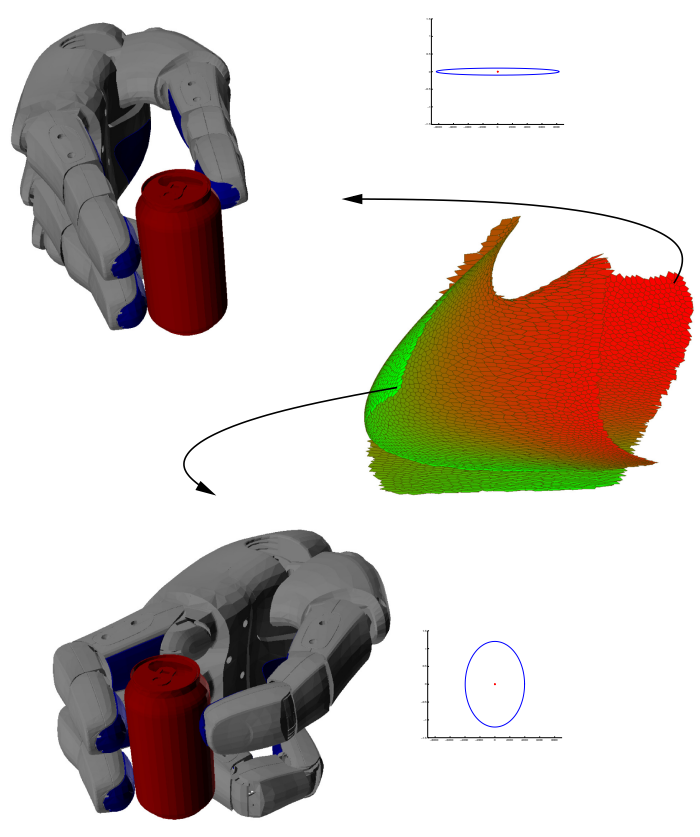

(a)

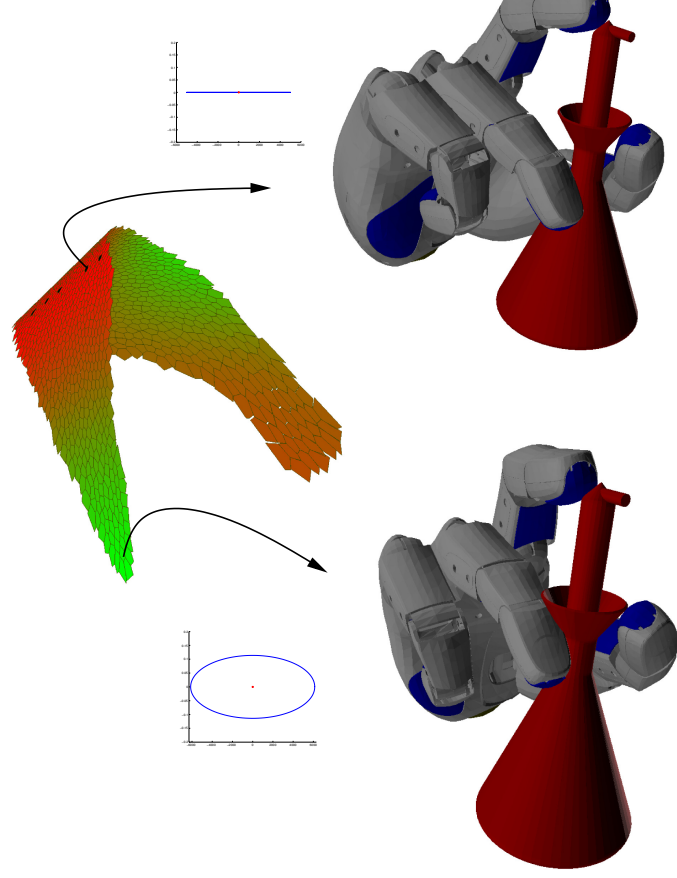

(b)

Fig. 10. Grasp optimization results for the Schunk anthropomorphic hand: (a) when holding a can and (b) holding an oil drizzler. In both cases the manipulability is evaluated using the volume of the manipulability ellipsoid. The atlases are shown at the center of the figure with green and red representing configuration with large and small manipulability, respectively, and black representing singular configurations. The top and bottom figures show the worst and the best configurations, respectively, with the associated projections of the manipulability ellipsoid.

of 13 , we keep more than $99 \%$ of the motion capability of the hand. In this case, the hand motions are computed from a database of human hand configurations [20]. The figure shows the results attending to the volume criterion. In this case, the atlas includes 4800 charts obtained with $r=1$ and $\epsilon=0.5$. The atlas is relatively small due to the joint limits. The worst and best grasps are shown in the top and bottom part of the figure, respectively. As depicted, the manipulability ellipsoid of the worst grasp is much smaller than that of the best one. As in the planar case, the manipulability is low when the fingers are almost extended. Notice that in this example local extrema are also present, hindering the obtention of good results with local optimization methods. The overall optimization takes 140 seconds to generate the atlas and 10 seconds to evaluate the manipulability criterion.

Figure 10p shows the results obtained when optimizing the grasp of a Marquina oil bottle. To comply with the task requirements, the index finger must contact the top of the bottle along a curve, and the two other fingers are constrained to contact patches in the middle and bottom sections of the bottle. Therefore, the fingers contact the object on disjoint patches with different sizes and orientations, which represents a general situation for the proposed approach. Due to the contact constraints, the number of variables is $n=142$, the dimension of $\mathcal{F}$ is $t=9$ and, thus, 7 constraints derived from the principal hand motions are used to obtain a set $\mathcal{R}$ of dimension $k=2$. The figure shows the obtained atlas, the best and worst grasps, and the associated manipulability ellipsoid projected as in the previous example. In this case, using the same parameters as before, the obtained atlas is smaller (1400 charts) resulting in a smaller processing time (45 seconds). The same problem can be optimized with $k=3$ simply by considering one less constraint derived from the principal hand motion analysis. In this case, the atlas includes 95000 charts and the optimization takes 1300 seconds. However, the optimal grasp is almost the same as that obtained with $k=2$. Thus, in this example, taking 6 principal hand motions is good enough to capture the relevant hand mobility.

\section{CONCLUSiOnS}

This paper has proposed a new procedure to obtain an optimal grasp satisfying a number of contact constraints between a robotic hand and an object. The procedure uses higherdimensional continuation tools to compute an atlas of the relevant grasp manifold. Each chart in this atlas parametrizes a portion of the manifold, and can be used to generate a grid of representative points to evaluate any quality index.

The procedure is global, in the sense that the grid spans the whole set of relevant grasps attainable from a given point, determining the optimal one up to the atlas resolution, without being trapped into local extrema. Moreover, the method is general as it can be applied to any hand structure, and to any desired quality index. The efficiency of the method critically depends on the dimension of the traced manifold. 
In the case of grasps, however, principal hand motions allow reducing the dimension of such manifold considerably. Note, that the proposed method keeps a large number of principal hand motions (up to 7 out of 13 for the Schunk hand), while previous methods [3, 20, 7] use a smaller number of them (typically 2). This is because the method integrates all contact constraints a priori, which already introduces a large dimension reduction.

The presented method operates in the connected component including an initial grasp. In most robotic hands, this is not an issue however, since, due to joint range limitations, the set of feasible grasps only contains one connected component. However, this might not be the case in general. We have to investigate ways to obtain one starting point in each connected component of the feasible grasp set. Finally, it would be interesting to generalize the method to deal with other contact models, including region-region contacts.

\section{ACKNOWLEDGMENTS}

This work has been partially supported by the Spanish Ministry of Science and Innovation under contracts DPI201018449 and DPI2010-15446. We would like to thank Patrick Grosch for providing the object models for the experiments.

\section{REFERENCES}

[1] A. Bicchi and D. Prattichizzo. Manipulability of Cooperating Robots with Unactuated Joints and ClosedChain Mechanisms IEEE Transactions on Robotics and Automation, 16(4):336-345, 2000.

[2] C. Borst, M. Fischer, and G. Hirzinger. Calculating hand configurations for precision and pinch grasps. In IEEE/RSJ International Conference on Intelligent Robots and System, pages 1553-1559, 2002.

[3] M. T. Ciocarlie and P. K. Allen. Hand Posture Subspaces for Dexterous Robotic Grasping. The International Journal of Robotics Research, 28(7):851-867, 2009.

[4] J. Denavit and R.S. Hartenberg. A kinematic notation for lower-pair mechanisms based on matrices. Transactions of the ASME. Journal of Applied Mechanics, 23:215-221, 1955.

[5] C. Fernández, O. Reinoso, A. Vicente, and R. Aracil. Kinematic Redundancy in Robot Grasp Synthesis. An Efficient Tree-based Representation. In IEEE International Conference on Robotics and Automation, pages 1203-1209, 2005.

[6] C. Ferrari and J. Canny. Planning optimal grasps. In IEEE International Conference on Robotics and Automation, pages 2290-2295, 1992.

[7] M. Gabiccini and A. Bicchi. On the Role of Hand Synergies in the Optimal Choice of Grasping Forces. In Proceedings of Robotics: Science and Systems, 2010.

[8] P. Gorce and N. Rezzoug. Grasping posture learning with noisy sensing information for a large scale of multifingered robotic systems. Journal of Robotic Systems, 22 (12):711-724, 2005.
[9] Y. Guan and H. Zhang. Kinematic feasibility analysis of 3-D multifingered grasps. IEEE Transactions on Robotics and Automation, 19(3):507-513, 2003.

[10] M. E. Henderson. Multiple Parameter Continuation: Computing Implicitly Defined k-Manifolds International Journal of Bifurcation and Chaos, 12(3):451-476, 2002.

[11] M. E. Henderson. Numerical continuation methods for dynamical systems: path following and boundary value problems, chapter Higher-Dimensional Continuation. Springer, 2007.

[12] N. Kamakura, M. Matsuo, H. Ishii, F. Mitsuboshi, and Y. Miura. Patterns of static prehension in normal hands The American Journal of Occupational Therapy, 34(7): 437-445, 1980.

[13] Z. Li and S. Sastry. Task-oriented optimal grasping by multifingered robot hands. IEEE Transactions of Robotics and Automation, 4(1):32-44, 1988.

[14] V.-D. Nguyen. Constructing Force- Closure Grasps The International Journal of Robotics Research, 7(3):3-16, 1988.

[15] R. Platt, A. H. Fagg, and R. A. Grupen. Null-space grasp control: theory and experiments IEEE Transactions on Robotics, 26:282-295, 2010.

[16] N. S. Pollard. Closure and Quality Equivalence for Efficient Synthesis of Grasps from Examples. The International Journal of Robotics Research, 23(6):595613, 2004

[17] M. Roa and R. Suárez. Computation of Independent Contact Regions for Grasping 3-D Objects IEEE Transactions on Robotics, 25(4):839-850, 2009.

[18] C. Rosales, L. Ros, J. M. Porta, and R. Suárez. Synthesizing Grasp Configurations with Specified Contact Regions. The International Journal of Robotics Research, 30(4):431-443, 2011.

[19] J. Rosell, X. Sierra, L. Palomo, and R. Suárez. Finding Grasping Configurations of a Dexterous Hand and an \begin{tabular}{l|l} 
Industrial Robot & In IEEE International Conference on
\end{tabular} Robotics and Automation, pages 1178-1183, 2005.

[20] J. Rosell, R. Suárez, C. Rosales, and A. Pérez. Autonomous motion planning of a hand-arm robotic system based on captured human-like hand postures. $A u$ tonomous Robots, pages 1-16 (online first), 2011.

[21] M. Santello, M. Flanders, and J. F. Soechting. Postural Hand Synergies for Tool Use. The Journal of Neuroscience, 18(23):10105-10115, 1998.

[22] J. C. Trinkle, A. O. Farahat, and P. F. Stiller. Firstorder stability cells of active multi-rigid-body systems IEEE Transactions on Robotics and Automation, 11(4): 545-557, 1995.

[23] C. Wampler and A. Morgan. Solving the 6R inverse position problem using a generic-case solution methodology Mechanism and Machine Theory, 26(1):91-106, 1991.

[24] K. Woelfl and F. Pfeiffer. Grasp strategies for a dextrous robotic hand. In IEEE/RSJ/GI International Conference on Intelligent Robots and Systems, pages 366-373, 1994. 